\title{
Effect of quantum statistics on the gravitational weak equivalence principle
}

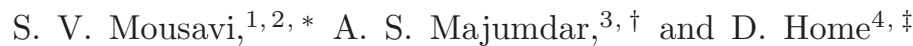 \\ ${ }^{1}$ Department of Physics, The University of Qom, Qom, Iran \\ ${ }^{2}$ School of Physics, Institute for Research in Fundamental Sciences (IPM), P.O.Box 19395-5531, Tehran, Iran \\ ${ }^{3} S$. N. Bose National Center for Basic Sciences, \\ Block JD, Sector III, Salt Lake, Kolkata 700 098, India \\ 4 CAPSS, Department of Physics, Bose Institute, Sector-V, Salt Lake, Kolkata 700 091, India
}

\begin{abstract}
We study the effect of quantum statistics on the arrival time distribution of quantum particles computed through the probability current density. It is shown that symmetrization or asymmetrization of the wave function affects the arrival time distribution for even freely propagating particles. In order to investigate the effect of statistics on the weak equivalence principle in quantum mechanics (WEQ), we then compute the mean arrival time for wave packets in free fall. The violation of WEQ through the effect of statistics on the mass-dependence of the mean arrival time is clearly exhibited. We finally evaluate the effect of spin on the violation of WEQ using a different approach by including an explicit spin-dependence in the probability current distribution, and compare it with the approach using particle statistics. Our results show WEQ re-emerges smoothly in the limit of large mass.
\end{abstract}

PACS numbers: 03.65.-w, 03.65.Ta

Keywords: Weak equivalence principle, Quantum statistics, Arrival time distribution, Spin

\section{INTRODUCTION}

The statistics of elementary particles is a key underlying ingredient of quantum field theory which has been most successful in explaining three, viz. electromagnetic, weak and strong nuclear forces, of the four fundamental forces in nature. On the other hand, the explicit role of quantum statistics in physical phenomena such as Bose Condensation [1] is also being increasingly displayed in recent years. Practical applications of such statistics is potentially wide, ranging from optical lattices [2] to quantum information [3]. In most practical manifestations of the role of Bose statistics, a crucial role is played by the measurement of the time of flight distribution [4]. For example, the coherence properties of a condensate of ultracold atoms is imprinted on the time of flight distribution when the trapping potential is released and the particles undergo free fall. Thus, the effect of gravity on the motion of quantum particles seems to play an important role in the inference of attributes related to the statistics obeyed by them.

The motion of freely falling particles is traditionally taken to conform to the weak equivalence principle (WEP) of gravitation which states that all sufficiently small objects fall with the same acceleration independent of their mass and constituent in a uniform gravitational field. WEP is regarded to be a fundamentally classical and local principle. Study of the equivalence principle in quantum mechanics has evoked enough interest. A statement of the principle in quantum mechanics is as follows: "The results of experiments in an external potential comprising just a sufficiently weak, homogeneous gravitational field, as determined by the wavefunction, are independent of the mass of the system" [5]. This assertion is also called the weak equivalence principle of quantum mechanics (WEQ).

Different approaches have been used to study the possibility of violation of weak equivalence principle in quantum mechanics, starting from the prediction of mass-dependence of the radii, frequencies and binding energy of a particle in an external gravitational field [6]. Experimental probes into possibility of violation of the weak equivalence principle in quantum mechanics have been attempted using interference phenomenon associated with the gravitational potential in neutron and atomic interferometry experiments [7, 8]. However, the phase shifts observed in such experiments 7] may be expressed in terms of directly observable quantities which turn out to be independent of mass [9], thus ruling out the violation in this context. Moreover, atomic interferometry experiments [8] need to be performed with different atomic species in order for a rigorous experimental test of the weak equivalence principle at the quantum level. A gedanken experiment studying the free fall of quantum test particles in a uniform gravitational field predicts mass-dependence of the time of flight distribution [10]. Another approach using a model quantum clock predicts

\footnotetext{
*Electronic address: vmousavi@qom.ac.ir

${ }^{\dagger}$ Electronic address: archan@bose.res.in

‡Electronic address: dhome@bosemain.boseinst.ac.in
} 
violation of WEQ in the vicinity of the turning point of classical trajectories 11. An explicit mass dependence of the position probabilities has been shown for quantum particles projected upwards against gravity around both the classical turning point and the point of initial projection using Gaussian [12] and non-Gaussian [13] wavepackets. Moreover, explicit mass dependence of the mean arrival time at an arbitrary detector location has also been predicted for a Gaussian [12] wave-packet under free fall. Such mass-dependence may be enhanced using suitably chosen nonGaussian wavepackets [13]. Thus, both the position probabilities and the mean arrival time show the violation of WEQ for quantum particles described by Gaussian and non-Gaussian wavepackets. It has been discussed violation of WEQ arises as a consequence of the spread of wave packets, the magnitude of which itself depends on the mass. An illustration of this effect has been presented based on Bohmian trajectories [13].

The above studies have established the violation of WEQ in single particle quantum mechanics. However, the effect of quantum statistics on the violation of WEQ has remained uninvestigated till date. As pointed out earlier, several important phenomena based on quantum statistics is experimentally revealed through the measurement of time of flight of quantum particles in free fall. Consideration of quantum mechanical effects on such time of flight distributions beyond the standard semi-classical analysis could reveal interesting observational effects, as has been debated earlier in the literature 14]. In the present work we are interested to examine the effect of quantum statistics on the WEQ. With the above motivation in this paper we study the effect of statistics on the arrival time distribution of a system of freely falling wavepackets consisting of two identical particles.

Our analysis is based on the probability current approach for computing the mean arrival time distribution of wavepackets [15]. Before embarking on the study of WEQ, we will first consider the free evolution of a system of two identical particles to investigate if there is any effect of Bose-Einstein (BE) or Fermi-Dirac (FD) statistics on the arrival time distributions. Quantum statistics is fundamentally related to the spin of the quantum particles. It has been shown earlier that inclusion of spin enables a unique determination of the relativistic probability current [16, 17] leading to an explicit spin-dependent effect at the non-relativistic level for freely evolving fermions [18] as well as bosons [19]. Here we present a calculation of the effect of such explicit spin-dependence on the violation of WEQ for a system of two identical particles in free fall.

The plan of this paper is as follows. In the next section we present the framework for studying the arrival time distribution for a system of two particles using the probability current approach. We show that symmetrization or anti-symmetrization of the wavefunction leads to differences from the case of Maxwell-Boltzmann statistics when there is overlap of the wavepackets. In section III the effect of BE and FD statistics on freely propagating wavepackets is studied. In section IV we address the main motivation of the paper by studying the violation of WEQ separately using two different schemes, viz, (a) effect of statistics on the arrival time distribution, and (b) effect of explicit spin-dependence on the arrival time distribution of freely falling wavepackets. Section V contains a summary of the main results along with certain concluding remarks.

\section{ARRIVAL TIME DISTRIBUTION FOR A TWO-PARTICLE SYSTEM}

Consider a two-body system composed of two non-interacting identical particles in an external field. Identical particles are classically distinguishable and obey Maxwell-Boltzmann (MB) statistics, while they are indistinguishable in quantum mechanics and obey different statistics. For Fermi-Dirac (Bose-Einstein) statistics the total wavefunction must be antisymmetrized (symmetrized) under the exchange of particles in the system. Since particles do not interact, solutions of the Schrödinger equation are constructed from two single-particle wavefunctions $\psi_{a}$ and $\psi_{b}$ as follows [5]:

$$
\begin{aligned}
\Psi_{\mathrm{MB}}\left(z_{1}, z_{2}, t\right) & =\psi_{a}\left(z_{1}, t\right) \psi_{b}\left(z_{2}, t\right), \\
\Psi_{ \pm}\left(z_{1}, z_{2}, t\right) & =N_{ \pm}\left[\psi_{a}\left(z_{1}, t\right) \psi_{b}\left(z_{2}, t\right) \pm \psi_{b}\left(z_{1}, t\right) \psi_{a}\left(z_{2}, t\right)\right],
\end{aligned}
$$

where upper (lower) sign stands for BE (FD) statistics and the normalization constants are given by $N_{ \pm}=[2(1 \pm$ $\left.\left.\left|\left\langle\psi_{a}(t) \mid \psi_{b}(t)\right\rangle\right|^{2}\right)\right]^{-1 / 2}$.

Two-body wavefunctions (21) are solutions of the two-body Schrödinger equation

$$
i \hbar \frac{\partial}{\partial t} \Psi\left(z_{1}, z_{2}, t\right)=\left(-\frac{\hbar^{2}}{2 m} \frac{\partial^{2}}{\partial z_{1}^{2}}-\frac{\hbar^{2}}{2 m} \frac{\partial^{2}}{\partial z_{2}^{2}}+V\left(z_{1}, t\right)+V\left(z_{2}, t\right)\right) \Psi\left(z_{1}, z_{2}, t\right),
$$

where the Hamiltonian is clearly symmetric under the exchange of $z_{1}$ and $z_{2}$.

The one-body probability density for observing a particle at point $z$ regardless of the position of the other particle, 
is given by [20]

$$
\begin{aligned}
\rho_{1}(z, t) & =\int d z_{1} d z_{2} \delta\left(z-z_{1}\right)\left|\Psi\left(z_{1}, z_{2}, t\right)\right|^{2}=\int d z_{2}\left|\Psi\left(z, z_{2}, t\right)\right|^{2} \\
& =\left|N_{ \pm}\right|^{2} \int d z_{2}\left|\psi_{a}(z, t) \psi_{b}\left(z_{2}, t\right) \pm \psi_{b}(z, t) \psi_{a}\left(z_{2}, t\right)\right|^{2} \\
& \equiv\left|N_{ \pm}\right|^{2}\left(\left|\psi_{a}(z, t)\right|^{2} \pm 2 \Re\left[\left\langle\psi_{a}(t) \mid \psi_{b}(t)\right\rangle \psi_{b}^{*}(z, t) \psi_{a}(z, t)\right]+\left|\psi_{b}(z, t)\right|^{2}\right),
\end{aligned}
$$

where $\left\langle\psi_{a}(t) \mid \psi_{b}(t)\right\rangle=\int d x \psi_{a}^{*}(x, t) \psi_{b}(x, t)$ is the overlap integral and the normalization condition for single-particle wavefunctions $\psi_{a}$ and $\psi_{b}$ has been used. From the unitarity property of the time evolution operator, $U(t, 0)=$ $e^{-i H t / \hbar}$, one sees that the overlap integral is independent of time, i.e., $\left\langle\psi_{a}(t) \mid \psi_{b}(t)\right\rangle=\left\langle\psi_{a}(0)\left|U^{\dagger}(t, 0) U(t, 0)\right| \psi_{b}(0)\right\rangle=$ $\left\langle\psi_{a}(0) \mid \psi_{b}(0)\right\rangle$.

The single-particle continuity equation may be derived [21] in the following way. The partial derivative of $\rho_{1}(z, t)$ with respect to the time is calculated from eq. (4) by performing integration by parts and using the fact that the wavefunction vanishes at infinity, to obtain

$$
\frac{\partial \rho_{1}(z, t)}{\partial t}=-\frac{\hbar}{m} \Im\left\{\frac{\partial}{\partial z} \int d z_{2} \Psi^{*}\left(z, z_{2}, t\right) \frac{\partial \Psi\left(z, z_{2}, t\right)}{\partial z}\right\}
$$

Now, using the normalization condition for the single-particle wavefunctions, one obtains the continuity equation 21]

$$
\frac{\partial \rho_{1}(z, t)}{\partial t}+\frac{\partial}{\partial z} j_{1}(z, t)=0
$$

for the one-body probability density $\rho_{1}(z, t)$ (4), with the one-body probability current density given by

$$
j_{1}(z, t)=\frac{\hbar}{m}\left|N_{ \pm}\right|^{2} \Im\left\{\psi_{a}^{*} \frac{\partial \psi_{a}}{\partial z}+\psi_{b}^{*} \frac{\partial \psi_{b}}{\partial z} \pm\left\langle\psi_{a}(t) \mid \psi_{b}(t)\right\rangle \psi_{b}^{*} \frac{\partial \psi_{a}}{\partial z} \pm\left\langle\psi_{b}(t) \mid \psi_{a}(t)\right\rangle \psi_{a}^{*} \frac{\partial \psi_{b}}{\partial z}\right\} .
$$

For distinguishable particles obeying classical MB statistics, each particle has its corresponding continuity equation,

$$
\frac{\partial \rho^{(i)}(z, t)}{\partial t}+\frac{\partial}{\partial z} j^{(i)}(z, t)=0
$$

with

$$
\rho^{(1)}(z, t)=\left|\psi_{a}(z, t)\right|^{2}, \quad \quad \rho^{(2)}(z, t)=\left|\psi_{b}(z, t)\right|^{2},
$$

and

$$
j^{(1)}(z, t)=\frac{\hbar}{m} \Im\left\{\psi_{a}^{*}(z, t) \frac{\partial \psi_{a}(z, t)}{\partial z}\right\}, \quad j^{(2)}(z, t)=\frac{\hbar}{m} \Im\left\{\psi_{b}^{*}(z, t) \frac{\partial \psi_{b}(z, t)}{\partial z}\right\} .
$$

So, for a two-particle system, the MB probability density and probability current density are given by

$$
\rho_{\mathrm{MB}}(z, t)=\frac{1}{2}\left(\rho^{(1)}(z, t)+\rho^{(2)}(z, t)\right), \quad j_{\mathrm{MB}}(z, t)=\frac{1}{2}\left(j^{(1)}(z, t)+j^{(2)}(z, t)\right)
$$

From eqs. (4) and (7), one sees that as long as the single-particle wavefunctions have negligible overlap, i.e., $\left\langle\psi_{a}(t) \mid \psi_{b}(t)\right\rangle \simeq 0$, there is no need for symmetrization. As a result, one can ignore indistinguishability of particles and thus use MB statistics for which motions of particles are independent. Hence, in the latter case a similar analysis to the one-body systems discussed earlier [12, 13] may be employed.

The probability current approach for computation of the arrival time distribution of quantum particles not only provides an unambiguous definition of arrival time at the quantum mechanical level [15 18], but also furnishes a way of obtaining the classical limit of the mean arrival time for massive quantum particles 22]. Here we employ the probability current approach to study the effect of particle statistics on the arrival time distribution of a two-body system. In this approach, the arrival time distribution at a detector location $z=Z$ is given by [15],

$$
\Pi(Z, t)=\frac{\left|j_{1}(Z, t)\right|}{\int_{0}^{\infty} d t\left|j_{1}(Z, t)\right|} .
$$


As a result, one obtains

$$
\tau(Z)=\int_{0}^{\infty} d t t \Pi(Z, t)
$$

for the mean arrival time at the detector location $z=Z$. It may be noted that the above expression for the mean arrival time follows uniquely [23] in a causal interpretation of quantum mechanics provided by the Bohmian model [24]. Note also that eqs.(12) and (13) represent the single-particle arrival time distribution and the mean arrival time, respectively, when the effects of particle statistics of the considered two-body system have been taken into account in the computation of the single-particle current density through the process of symmetrization or asymmetrization of

the wavefunction. In the following analysis we will consider the initial single particle wavefunctions to be Gaussians given by

$$
\psi_{i}(z, 0)=\left(2 \pi \sigma_{0 i}^{2}\right)^{-1 / 4} \exp \left[i k_{i} z-\frac{\left(z-z_{c i}\right)^{2}}{4 \sigma_{0 i}^{2}}\right], \quad i=a, b
$$

and then compute the arrival time distribution of the cases of first free evolution, and then free fall under gravity. In eq.(14), $\sigma_{0 i}, z_{c i}$ and $k_{i}$ represent respectively the rms width, center and kick momentum of the i-th packet.

\section{EFFECT OF STATISTICS ON FREELY PROPAGATING WAVEPACKETS}

In this section we study the effect of symmetrization and asymmetrization of a two-body freely propagating wavefunction on the arrival time distribution computed through the probability current approach. The initial one-particle wavefunctions are taken to be Gaussian packets given by eq.(14). The time evolved wave functions are obtained to be

$$
\psi_{i}(z, t)=\frac{1}{\left(2 \pi s_{t i}^{2}\right)^{1 / 4}} \exp \left\{\frac{i m}{2 \hbar t}\left[\left(z^{2}+i \frac{\hbar t}{2 m \sigma_{0 i}^{2}} z_{c i}^{2}\right)-\frac{\sigma_{0 i}}{s_{t i}}\left(z-z_{c i}-\frac{\hbar t}{m} k_{i}+\frac{s_{t i}}{\sigma_{0 i}} z_{c i}\right)^{2}\right]\right\}
$$

where $s_{t i}=\sigma_{0 i}\left(1+i \hbar t / 2 m \sigma_{0 i}^{2}\right)$ denotes the time-evolved spread of the wavepackets. The overlap integral is given by

$$
\left\langle\psi_{a}(t) \mid \psi_{b}(t)\right\rangle=\sqrt{\frac{2 \sigma_{0 a} \sigma_{0 b}}{\sigma_{0 a}^{2}+\sigma_{0 b}^{2}}} \exp \left[-\frac{4\left(k_{a}-k_{b}\right)^{2} \sigma_{0 a}^{2} \sigma_{0 b}^{2}+\left(z_{c a}-z_{c b}\right)^{2}+4 i\left(k_{a}-k_{b}\right)\left(\sigma_{0 b}^{2} z_{c a}+\sigma_{0 a}^{2} z_{c b}\right)}{4\left(\sigma_{0 a}^{2}+\sigma_{0 b}^{2}\right)}\right],
$$

and thus, the normalization constants read

$$
N_{ \pm}=\frac{1}{\sqrt{2}}\left\{1 \pm \frac{2 \sigma_{0 a} \sigma_{0 b}}{\sigma_{0 a}^{2}+\sigma_{0 b}^{2}} \exp \left[-\frac{4\left(k_{a}-k_{b}\right)^{2} \sigma_{0 a}^{2} \sigma_{0 b}^{2}+\left(z_{c a}-z_{c b}\right)^{2}}{2\left(\sigma_{0 a}^{2}+\sigma_{0 b}^{2}\right)}\right]\right\}^{-1 / 2}
$$

One sees that the magnitude of the overlap integral decreases exponentially with the separation of the initial wavepackets. As the separation grows, the quantum and classical results approach:

$$
\begin{aligned}
& \rho_{1}(z, t) \simeq \frac{1}{2}\left(\left|\psi_{a}(z, t)\right|^{2}+\left|\psi_{b}(z, t)\right|^{2}\right)=\rho_{\mathrm{MB}}(z, t), \\
& j_{1}(z, t) \simeq \frac{1}{2} \frac{\hbar}{m} \Im\left\{\psi_{a}^{*} \frac{\partial \psi_{a}}{\partial z}+\psi_{b}^{*} \frac{\partial \psi_{b}}{\partial z}\right\}=j_{\mathrm{MB}}(z, t) .
\end{aligned}
$$

The position expectation value with respect to $\rho_{1}(z, t)$ reads

$$
\begin{aligned}
\langle z\rangle & =\int z \rho_{1}(z, t) d z \\
& =\left|N_{ \pm}\right|^{2}\left\{z_{\mathrm{cl}, a}+z_{\mathrm{cl}, b} \pm\left|\left\langle\psi_{a}(t) \mid \psi_{b}(t)\right\rangle\right|^{2}\left(\frac{\hbar t\left(k_{a} \sigma_{0 a}^{2}+k_{b} \sigma_{0 b}^{2}\right)-m\left(z_{c a} \sigma_{0 a}^{2}+z_{c b} \sigma_{0 b}^{2}\right)}{m\left(\sigma_{0 a}^{2}+\sigma_{0 b}^{2}\right)}\right)\right\}
\end{aligned}
$$

where $z_{\mathrm{cl}, a}$ denotes the classical path of the center of the wavepacket $\psi_{a}$, i.e., $z_{\mathrm{cl}, a}=z_{c a}+\hbar k_{a} t / m$. One sees that the expectation value of the center of mass operator is just the position expectation value with respect to $\rho_{1}(z, t)$, i.e.,

$$
\begin{aligned}
\left\langle z_{\mathrm{cm}}\right\rangle(t) & =\left\langle\frac{z_{1}+z_{2}}{2}\right\rangle(t)=\iint d z_{1} d z_{2} \frac{z_{1}+z_{2}}{2}\left|\Psi\left(z_{1}, z_{2}, t\right)\right|^{2} \\
& =\frac{1}{2}\left\{\int d z_{1} z_{1} \int d z_{2}\left|\Psi\left(z_{1}, z_{2}, t\right)\right|^{2}+\int d z_{2} z_{2} \int d z_{1}\left|\Psi\left(z_{1}, z_{2}, t\right)\right|^{2}\right\}=\int d z z \rho_{1}(z, t) .
\end{aligned}
$$



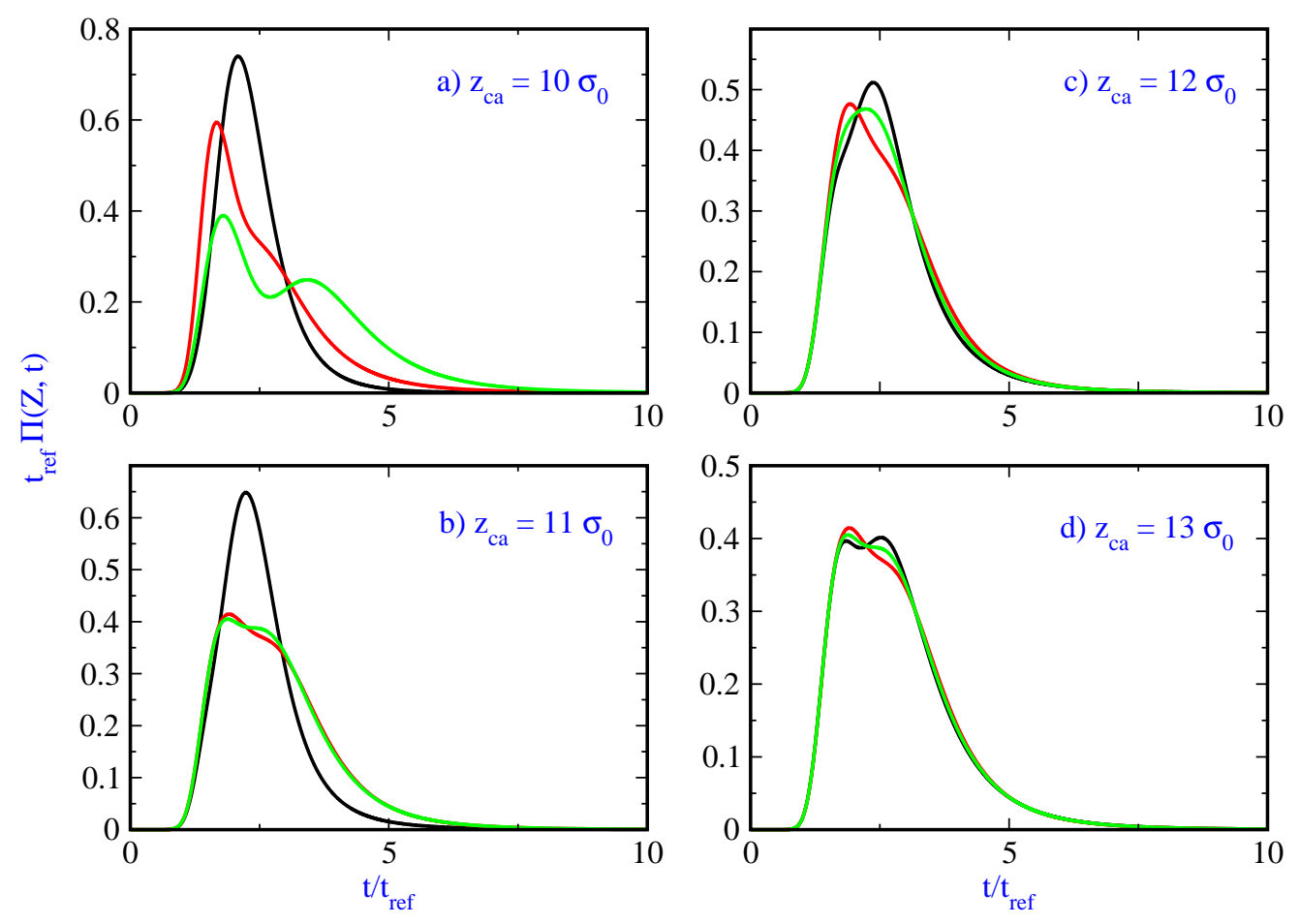

FIG. 1: (Color online) Arrival time distribution at the detector location $Z=0$ for different initial separation of the wavepackets for the free evolution case. Black curves stand for BE statistics, the red ones are for the FD statistics and the green ones show MB statistics. Here, $k_{a}=k_{b}=-2 / \sigma_{0}, z_{c b}=8 \sigma_{0}, \sigma_{0}=10 \mu \mathrm{m}, m=m_{n}$ and $t_{\mathrm{ref}}=2 m_{\mathrm{n}} \sigma_{0}^{2} / \hbar=3.165 \mathrm{~ms}$.

We calculate the arrival time distribution at the detector location $Z=0$ by substituting eq. (15) in the expression for the probability current given by eq.(17), and then substituting the obtained expression in eq.(12). The values of the parameters used for our numerical computations are given by: $\sigma_{0 a}=\sigma_{0 b}=\sigma_{0}=10 \mu \mathrm{m}, z_{c a}=10 \sigma_{0}, z_{c b}=8 \sigma_{0}$, $k_{a}=k_{b}=-2 / \sigma_{0}, m=m_{\mathrm{n}}=1.67 \times 10^{-27} \mathrm{~kg}$, and $t_{\mathrm{ref}}=\frac{2 m_{\mathrm{n}} \sigma_{0}^{2}}{\hbar}=3.165 \mathrm{~ms}$. In figure 1 the arrival time distribution has been plotted for different values of initial separation of the wavepackets. It is observed that the inclusion of particle statistics through symmetrization and antisymmetrization of a two-body wavefunction has a perceptible impact on the arrival time distribution. Such an effect of particle statistics on arrival times of freely propagating particles has been studied earlier by using the concept of the crossing state and the second quantization formalism [25]. In the present work we further compute the effect of initial separation of the overlapping wavepackets, as is displayed in the Figure 1. We fix the center of $\psi_{b}$ and change the center of $\psi_{a}$ from one plot to the other. Using eq. (16) and eq. (13), for the parameters of Fig. 1 one obtains

\begin{tabular}{||c|c|c|c|c||}
\hline$z_{c a} / \sigma_{0}$ & $\left\langle\psi_{a} \mid \psi_{b}\right\rangle$ & $\tau_{B E} / t_{r e f}$ & $\tau_{F D} / t_{r e f}$ & $\tau_{M B} / t_{r e f}$ \\
\hline 10 & 0.6065 & 2.371 & 2.546 & 2.427 \\
\hline 11 & 0.3247 & 2.518 & 2.614 & 2.561 \\
\hline 12 & 0.1353 & 2.682 & 2.707 & 2.694 \\
\hline 13 & 0.04394 & 2.826 & 2.829 & 2.822 \\
\hline
\end{tabular}

One sees that as the separation of the initial wavepackets grows, the quantum and classical results approach as a result of decrement of the overlap integral. 


\section{VIOLATION OF THE WEAK EQUIVALENCE PRINCIPLE OF QUANTUM MECHANICS}

\section{A. Effect of statistics on freely falling wavepackets}

The arrival time of freely falling wavepackets using the probability current approach has been studied earlier [12, 13], where it was noted that the the arrival time distribution acquires a mass-dependence due to wavepacket spread. In the previous section we have seen that the effect of particle statistics impacts the arrival time distribution even for the case of free evolution. Now, let us study the effect of symmetrization and asymmetrization of the wavefunction of a system of two particles falling freely under gravity. Here again we choose the initial single-particle wavefunctions as Gaussians given by eq.(14), and set the initial velocity of the particles to be zero, i.e., the particles are dropped from rest with $k_{a}=k_{b}=0$, and accelerate downwards under gravity with $g=10 \mathrm{~m} / \mathrm{s}^{2}$. All numerical calculations have been done based on the approximate value $g=10 \mathrm{~m} / \mathrm{s}^{2}$, since using the exact value of $g$ is not important for the arguments and reasoning. The time-evolved single-particle wavefunctions in the uniform gravitational field $V(z)=m g z$ are given by,

$$
\psi_{i}(z, t)=\frac{1}{\left(2 \pi s_{t i}^{2}\right)^{1 / 4}} \exp \left\{\frac{i m}{2 \hbar t}\left[\left(z^{2}-g t^{2} z-\frac{g^{2} t^{4}}{12}+i \frac{\hbar t}{2 m \sigma_{0 i}^{2}} z_{c i}^{2}\right)-\frac{\sigma_{0 i}}{s_{t i}}\left(z-z_{c i}+\frac{g t^{2}}{2}+\frac{s_{t i}}{\sigma_{0 i}} z_{c i}\right)^{2}\right]\right\}
$$

where $s_{t i}=\sigma_{0 i}\left(1+i \hbar t / 2 m \sigma_{0 i}^{2}\right)$. The overlap integral is given by,

$$
\left\langle\psi_{a}(t) \mid \psi_{b}(t)\right\rangle=\sqrt{\frac{2 \sigma_{0 a} \sigma_{0 b}}{\sigma_{0 a}^{2}+\sigma_{0 b}^{2}}} \exp \left[-\frac{\left(z_{c a}-z_{c b}\right)^{2}}{4\left(\sigma_{0 a}^{2}+\sigma_{0 b}^{2}\right)}\right],
$$

and hence, the normalization constants become

$$
N_{ \pm}=\frac{1}{\sqrt{2}}\left\{1 \pm \frac{2 \sigma_{0 a} \sigma_{0 b}}{\sigma_{0 a}^{2}+\sigma_{0 b}^{2}} \exp \left[-\frac{\left(z_{c a}-z_{c b}\right)^{2}}{2\left(\sigma_{0 a}^{2}+\sigma_{0 b}^{2}\right)}\right]\right\}^{-1 / 2}
$$

One sees that both overlap integral and normalization constants are independent of $g$. Here again the magnitude of the overlap integral decreases exponentially with the separation of the initial wavepackets, and as in the case of freely evolving wavepackets, the quantum results of the probability density and current approach the classical expressions as the separation grows. The position expectation value is given by

$$
\begin{aligned}
\langle z\rangle_{ \pm} & =\int z \rho_{1}(z, t) d z \\
& =\left|N_{ \pm}\right|^{2}\left\{z_{\mathrm{cl}, a}+z_{\mathrm{cl}, b} \pm\left|\left\langle\psi_{a}(t) \mid \psi_{b}(t)\right\rangle\right|^{2}\left(\frac{-g t^{2}}{2}-\frac{z_{c a} \sigma_{0 a}^{2}+z_{c b} \sigma_{0 b}^{2}}{\sigma_{0 a}^{2}+\sigma_{0 b}^{2}}\right)\right\}
\end{aligned}
$$

where $z_{\mathrm{cl}, a}$ shows the classical path of the center of the wavepacket $\psi_{a}$, i.e, $z_{\mathrm{cl}, a}=z_{c a}-g t^{2} / 2$. The position expectation value with respect to $\rho_{1}(z, t)$ is the expectation value of the center of mass operator. The quantity $\langle z\rangle_{ \pm}$is independent of mass.

For the free fall case and for $\sigma_{0 a}=\sigma_{0 b}=\sigma_{0}$ one obtains

$$
\begin{aligned}
\Delta z_{ \pm}(t) & =\sqrt{\int d z z^{2} \rho_{1}(z, t)-\left(\int d z z \rho_{1}(z, t)\right)^{2}} \\
& =\sigma_{0} \sqrt{\left(1+\frac{\hbar^{2} t^{2}}{4 m^{2} \sigma_{0}^{4}}\right)\left\{1 \pm \frac{\left(z_{c a}-z_{c b}\right)^{2}}{4 \sigma_{0}^{2}\left(\mp 1-\exp \left[\frac{\left(z_{c a}-z_{c b}\right)^{2}}{4 \sigma_{0}^{2}}\right]\right)}\right\}+\frac{\left(z_{c a}-z_{c b}\right)^{2}}{4}}
\end{aligned}
$$

for the width of the single-particle distribution. One clearly sees this quantity is independent of the gravity strength $g$ and its growth with time is slow for large masses.

We compute the arrival time distribution at the detector location $Z=0$ by substituting the expression for the timeevolved wavefunction eq.(21) in the expression for the probability current given by eq.(7), and then using eq.(12). Using the three quantities $\hbar, g$ and $\sigma_{0}$, one can construct a quantity $m_{0}=\hbar / \sqrt{g \sigma_{0}^{3}}$ with the dimension of mass, which has the value $m_{0}=1.055 \times 10^{-27} \mathrm{~kg} \approx m_{n}$, the neutron mass. We use $m_{n}$ to make the mass $m$ dimensionless. The values of the parameters used for our numerical calculations are given by: $\sigma_{0 a}=\sigma_{0 b}=\sigma_{0}=10 \mu \mathrm{m}, z_{c a}=10 \sigma_{0}$, 

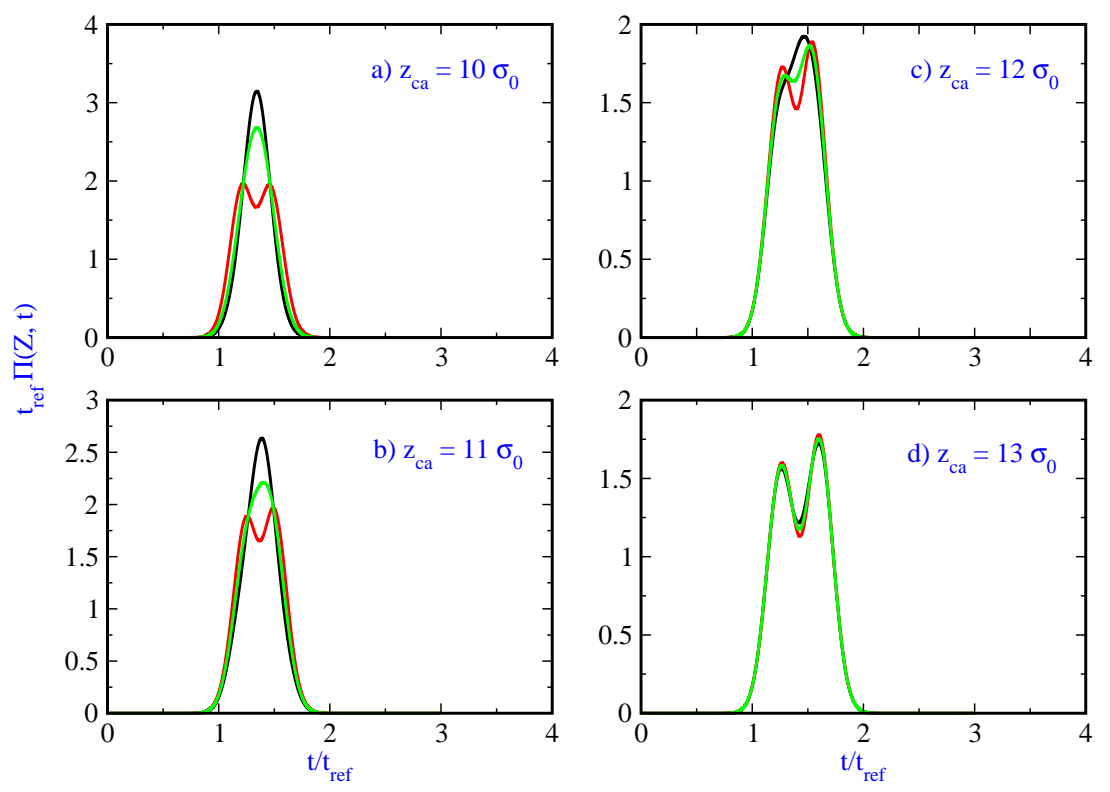

FIG. 2: (Color online) Arrival time distribution at the detector location $Z=0$ for different initial separation of the wavepackets for the free fall case. Black curves show BE statistics, the red ones are for the FD statistics and the green ones show MB statistics. Here, $z_{c b}=8 \sigma_{0}, \sigma_{0}=10 \mu \mathrm{m}, g=10 \mathrm{~m} / \mathrm{s}^{2}, m=m_{n}$ and $t_{\text {ref }}=2 m_{\mathrm{n}} \sigma_{0}^{2} / \hbar=3.165 \mathrm{~ms}$.

$z_{c b}=8 \sigma_{0}, m=m_{\mathrm{n}}=1.67 \times 10^{-27} \mathrm{~kg}$, and $t_{\mathrm{ref}}=\frac{2 m_{\mathrm{n}} \sigma_{0}^{2}}{\hbar}=3.165 \mathrm{~ms}$. In figure 2 the arrival time distribution has been plotted for different values of initial separation of the wavepackets. In this case too the particle statistics has an impact on the arrival time distribution. Using eq. (22) and eq. (13), for the parameters of Fig. 2 one obtains

\begin{tabular}{||c|c|c|c|c||}
\hline$z_{c a} / \sigma_{0}$ & $\left\langle\psi_{a} \mid \psi_{b}\right\rangle$ & $\tau_{B E} / t_{\text {ref }}$ & $\tau_{F D} / t_{\text {ref }}$ & $\tau_{M B} / t_{\text {ref }}$ \\
\hline 10 & 0.6065 & 1.339 & 1.341 & 1.340 \\
\hline 11 & 0.3247 & 1.374 & 1.375 & 1.374 \\
\hline 12 & 0.1353 & 1.407 & 1.407 & 1.407 \\
\hline 13 & 0.04394 & 1.439 & 1.439 & 1.439 \\
\hline
\end{tabular}

Such an effect of symmetrization and asymmetrization of a two-body wavefunction on arrival times of freely falling wavepackets may be regarded as nonlocal (in the sense that the single-particle arrival time distribution depends on the spatially separated second particle, as well), and thus contrary to the tenet of the local weak equivalence principle of classical gravity, which forms the inspiration of the statement of WEQ.

Now, in order to show the explicit mass dependence of arrival time for particles in free fall we plot the mean arrival time versus mass in the figure 3. Here again, the mean arrival time is computed at the detector location $Z=0$ using eq.(12) and eq.(13) after substituting the expression for the time-evolved wavefunction eq.(21) in the expression for the probability current given by eq.(7). The values of the parameters used are as before. Curves with label $\psi_{a}\left(\psi_{b}\right)$ show mean arrival time for wavepackets $\psi_{a}\left(\psi_{b}\right)$ respectively. The MB mean arrival time is given by $\tau_{\mathrm{MB}}=\frac{\tau_{a}+\tau_{b}}{2}$. One sees that for all types of statistics the mean arrival time decreases with mass at first and then becomes constant for large mass. The effect of symmetrization and asymmetrization of the wavefunction has a perceptible effect on the magnitude of the violation of WEQ. However, the violation of WEQ observed explicitly for low masses tends to disappear smoothly in the limit of large mass. Hence, even with the inclusion of particle statistics, the classical limit emerges smoothly through this approach.

One sees each of the three statistics gives a different mean arrival time in the large mass limit. The difference between the mean arrival times of bosons and fermions is related to the difference between the two quantities (i) position expectation value, $\langle z\rangle(t)$, with respect to the one-body probability density $\rho_{1}(z, t)$ and (ii) spread in position, 

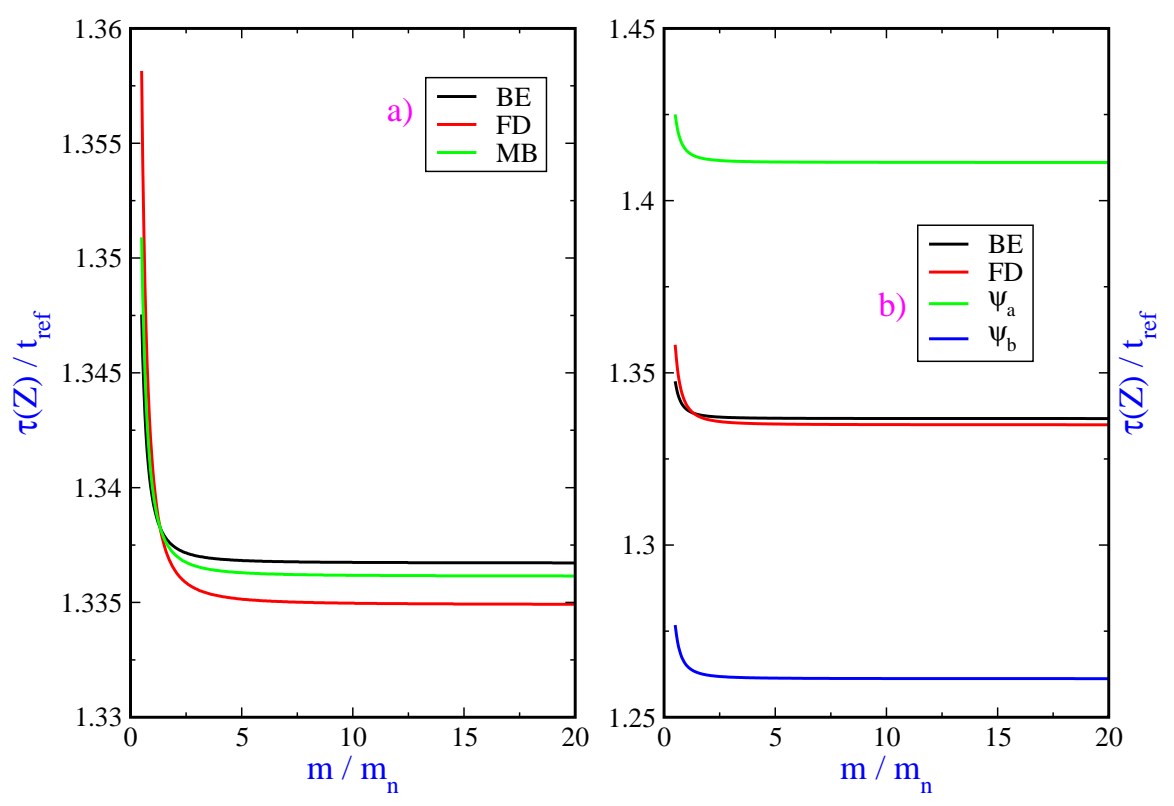

FIG. 3: (Color online) Mean arrival time at the detector location $Z=0$ versus mass for the free fall case. Here, $k_{a}=k_{b}=0$, $z_{c a}=10 \sigma_{0}, z_{c b}=8 \sigma_{0}, \sigma_{0}=10 \mu \mathrm{m}$ and $t_{\mathrm{ref}}=2 m_{\mathrm{n}} \sigma_{0}^{2} / \hbar=3.165 \mathrm{~ms}$. In b) we have included mean arrival time of single-particle systems which are described by wavepackets $\psi_{a}$ and $\psi_{b}$, i.e., $\tau_{a}$ and $\tau_{b}$.

$\Delta z(t)=\sqrt{\left\langle z^{2}\right\rangle-\langle z\rangle^{2}}$. At time $t=t_{ \pm}$,

$$
t_{ \pm}=\sqrt{\frac{z_{c a}+z_{c b}}{g} \frac{2 \mp \exp \left[-\frac{\left(z_{c a}-z_{c b}\right)^{2}}{4 \sigma_{0}^{2}}\right]}{2 \pm \exp \left[-\frac{\left(z_{c a}-z_{c b}\right)^{2}}{4 \sigma_{0}^{2}}\right]}} .
$$

the center of the one-body probability density $\rho_{1}(z, 0)$ arrives at the ground, the detector location. We have plotted $\langle z\rangle_{ \pm}(0)$ and $\Delta z_{ \pm}(t \pm)$ as a function of mass in Fig. 4 . As this figure shows $\Delta z_{+}\left(t_{+}\right)$and $\Delta z_{-}\left(t_{-}\right)$corresponding to bosonic and fermionic statistics, respectively, do not approach each other even in the large mass limit.

\section{B. Spin-dependent arrival time distribution}

In this subsection we discuss the effect of explicit inclusion of spin in the probability current density on the arrival time distribution of particles in free fall. It has been noted earlier [17] that in nonrelativistic quantum mechanics the form of the probability current density is not unique. It was shown [16] that the Dirac equation implies a unique expression for the probability current for spin- $1 / 2$ particles in the nonrelativistic limit. Uniqueness of the probability current is a generic consequence of any relativistic quantum dynamics. Effects of spin on the arrival time distribution for freely evolving wavepackets have been computed for fermions [18] as well as for bosons [19]. Here we obtain the spin-dependent arrival time distribution for a symmetric two-dimensional Gaussian wavepacket in order to compare the effect of explicit presence of spin in the probability current density with the effect of particle statistics for wavepackets falling freely under gravity. Taking into account the spin contribution to the probability current density for particles with spin- $1 / 2$, one obtains [16]

$$
\begin{aligned}
\mathbf{j}(\mathbf{x}, t ; \hat{\mathbf{s}}) & =\mathbf{j}_{\mathrm{Sch}}(\mathbf{x}, t)+\frac{1}{m} \nabla\left[\psi^{*}(\mathbf{x}, t) \psi(\mathbf{x}, t)\right] \times \mathbf{s} \\
& =\frac{\hbar}{m}\left\{\Im\left[\psi^{*}(\mathbf{x}, t) \nabla \psi(\mathbf{x}, t)\right]+\Re\left[\psi^{*}(\mathbf{x}, t) \nabla \psi(\mathbf{x}, t)\right] \times \hat{\mathbf{s}}\right\}
\end{aligned}
$$



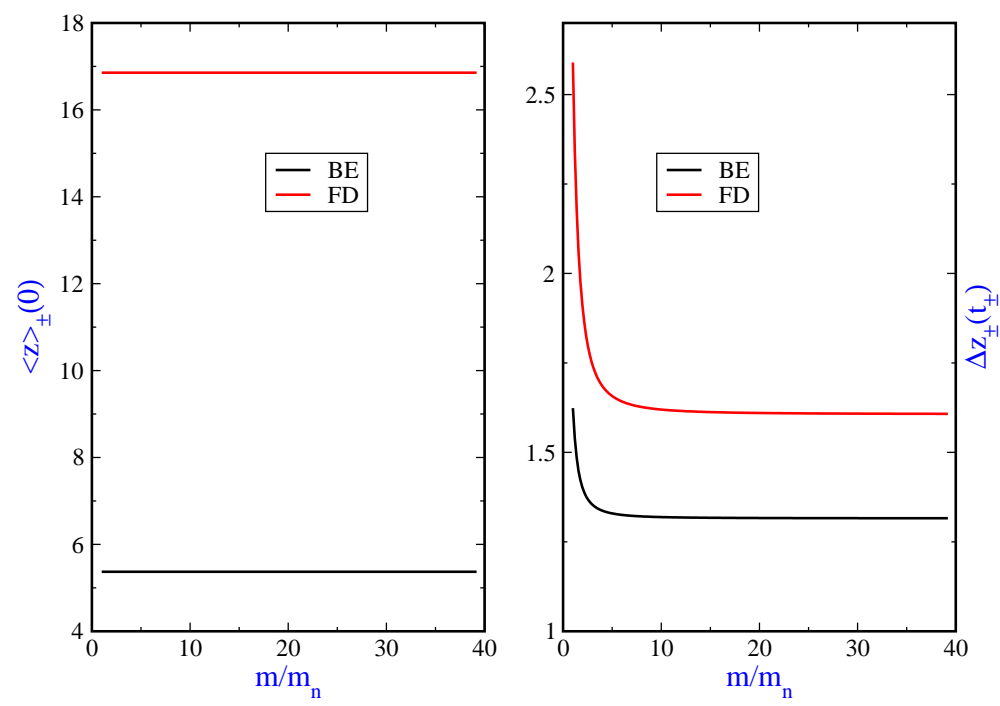

FIG. 4: (Color online) $\langle z\rangle_{ \pm}(0)$ and $\Delta z_{ \pm}\left(t_{ \pm}\right)$for the free fall case. Black curve shows BE statistics and the red one is for the FD statistics. Here, $z_{c a}=10 \sigma_{0} z_{c b}=8 \sigma_{0}, \sigma_{0}=10 \mu \mathrm{m}$ and $g=10 \mathrm{~m} / \mathrm{s}^{2}$.

for a spin eigenstate $\chi$ in the absence of a magnetic field. Here, $\mathbf{j}_{\mathrm{Sch}}(\mathbf{x}, t)$ is the usual Schrödinger current, whereas the second term denotes the spin-dependent contribution with $\mathbf{s}=\frac{\hbar}{2} \hat{\mathbf{s}}=\frac{\hbar}{2} \chi^{\dagger} \hat{\sigma} \chi$. For a two-dimensional system with a wavefunction which is in the factorized form $\psi(\mathbf{x}, t)=\psi_{x}(x, t) \psi_{z}(z, t)$, the probability current density takes the form

$$
\begin{aligned}
j_{x}(x, z, t ; \hat{\mathbf{s}}) & =\frac{\hbar}{m}\left\{\left|\psi_{z}(z, t)\right|^{2} \Im\left[\psi_{x}^{*}(x, t) \partial_{x} \psi_{x}(x, t)\right]-\left|\psi_{x}(x, t)\right|^{2} \Re\left[\psi_{z}^{*}(z, t) \partial_{z} \psi_{z}(z, t)\right]\right\}, \\
j_{z}(x, z, t ; \hat{\mathbf{s}}) & =\frac{\hbar}{m}\left\{\left|\psi_{x}(x, t)\right|^{2} \Im\left[\psi_{z}^{*}(z, t) \partial_{z} \psi_{z}(z, t)\right]+\left|\psi_{z}(z, t)\right|^{2} \Re\left[\psi_{x}^{*}(x, t) \partial_{x} \psi_{x}(x, t)\right]\right\},
\end{aligned}
$$

for $\hat{\mathbf{s}}=(0,1,0)$. In the uniform gravitational field $V(z)=m g z$, by taking the initial wavefunction as a two-dimensional Gaussian wavepacket,

$$
\psi_{0}(x, z)=\frac{1}{\sqrt{2 \pi} \sigma_{0}} \exp \left\{-\frac{x^{2}}{4 \sigma_{0}^{2}}-\frac{\left(z-z_{c}\right)^{2}}{4 \sigma_{0}^{2}}+i k_{0} z\right\},
$$

the modulus of the Schrödinger current takes the form

$$
\begin{aligned}
\left|\mathbf{j}_{\mathrm{Sch}}(x, z, t)\right| & =\frac{\sqrt{f_{4}(t) m^{4}+f_{3}(t) m^{3}+f_{2}(z, t) m^{2}+f_{1}(z, t) m+f_{0}(x, z, t)}}{16 \pi m^{2} \sigma_{0}^{2} \sigma_{t}^{4}} \\
& \times \exp \left\{-\frac{x^{2}+\left[z-z_{\mathrm{cl}}(t)\right]^{2}}{2 \sigma_{t}^{2}}\right\},
\end{aligned}
$$

while the modulus of the total spin-dependent probability current density is given by

$$
|\mathbf{j}(x, z, t ; \hat{\mathbf{s}})|=\frac{\sqrt{h_{2}(t) m^{2}+h_{1}(x, t) m+h_{0}(x, z, t)}}{8 \pi m \sigma_{0} \sigma_{t}^{3}} \exp \left\{-\frac{x^{2}+\left[z-z_{\mathrm{cl}}(t)\right]^{2}}{2 \sigma_{t}^{2}}\right\},
$$

where,

$$
\begin{aligned}
f_{0}(x, z, t) & =\hbar^{4} t^{2}\left\{g^{2} t^{4}+4\left[x^{2}+\left(z-z_{c}\right)^{2}\right]+4 g t^{2}\left(-z+z_{c}\right)\right\}, \\
f_{1}(z, t) & =-16 \hbar^{3} k_{0} \sigma_{0}^{4} t\left(g t^{2}-2 z+2 z_{c}\right) \\
f_{2}(z, t) & =16 \hbar^{2} \sigma_{0}^{4}\left[4 k_{0}^{2} \sigma_{0}^{4}+g t^{2}\left(g t^{2}-2 z+2 z_{c}\right)\right], \\
f_{3}(t) & =-128 g \hbar k_{0} \sigma_{0}^{8} t \\
f_{4}(t) & =64 g^{2} \sigma_{0}^{8} t^{2}
\end{aligned}
$$

and

$$
\begin{aligned}
h_{0}(x, z, t) & =\hbar^{2}\left\{16 k_{0}^{2} \sigma_{0}^{4}+g^{2} t^{4}-16 k_{0} \sigma_{0}^{2} x+4\left[x^{2}+\left(z-z_{c}\right)^{2}\right]+4 g t^{2}\left(-z+z_{c}\right)\right\}, \\
h_{1}(x, t) & =16 g \hbar \sigma_{0}^{2} t\left(-2 k_{0} \sigma_{0}^{2}+x\right), \\
h_{2}(t) & =16 g^{2} \sigma_{0}^{4} t^{2} .
\end{aligned}
$$



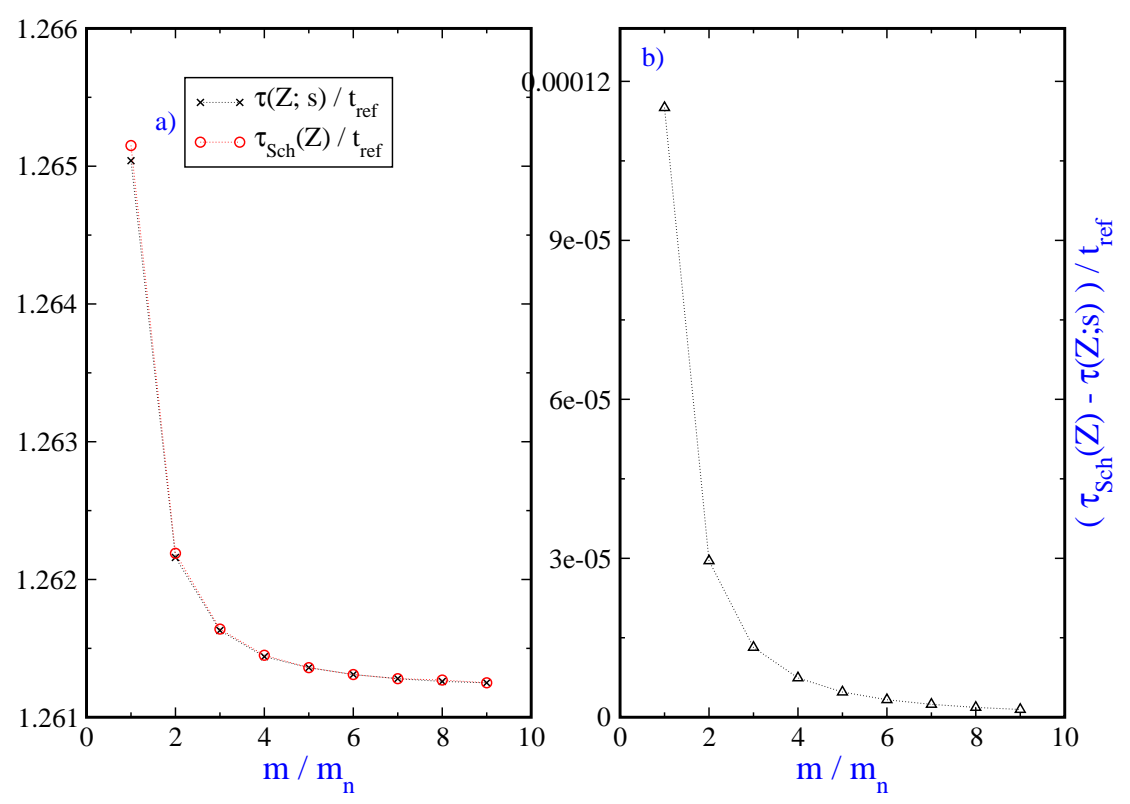

FIG. 5: (Color online) a) Spin-dependent (black points) and Spin-independent (red points) mean arrival time at the detector plane $Z=0$ and b) their difference, versus mass for the free fall of the 2D symmetric Gaussian packet (31). The parameters of the falling wavepacket have been chosen as $z_{c}=8 \sigma_{0}$ and $\sigma_{0}=10 \mu \mathrm{m}, m_{\mathrm{n}}=1.67 \times 10^{-27} \mathrm{~kg}$ and $t_{\mathrm{ref}}=2 \mathrm{~m}_{n} \sigma_{0}^{2} / \hbar=3.161 \mathrm{~ms}$.

In eqs. (32) and (33), $\sigma_{t}=\sigma_{0} \sqrt{1+\frac{\hbar^{2} t^{2}}{4 m^{2} \sigma_{0}^{4}}}$ and $z_{\mathrm{cl}}(t)=-\frac{1}{2} g t^{2}+u_{z} t+z_{c}$ with $u_{z}=\hbar k_{0} / m$.

In order to obtain the arrival time distribution at the plane $z=Z$ we integrate over the space coordinate $x$. Thus, the normalized arrival time distribution is given by

$$
\Pi_{\text {Sch }}(Z, t)=\frac{\int_{-\infty}^{\infty} d x\left|\mathbf{j}_{\operatorname{Sch}}(x, Z, t)\right|}{\int_{0}^{\infty} d t \int_{-\infty}^{\infty} d x\left|\mathbf{j}_{\operatorname{Sch}}(x, Z, t)\right|}
$$

in the absence of spin contribution, while it reads

$$
\Pi(Z, t ; \hat{\mathbf{s}})=\frac{\int_{-\infty}^{\infty} d x|\mathbf{j}(x, Z, t ; \hat{\mathbf{s}})|}{\int_{0}^{\infty} d t \int_{-\infty}^{\infty} d x|\mathbf{j}(x, Z, t ; \hat{\mathbf{s}})|},
$$

considering the spin effect. We have integrated over $x$ from $-\infty$ to $\infty$ to compare the results with the corresponding one-dimensional system. The corresponding mean arrival times are respectively given by

$$
\begin{aligned}
\tau_{\mathrm{Sch}}(Z) & =\int_{0}^{\infty} d t t \Pi_{\mathrm{Sch}}(Z, t), \\
\tau(Z ; \hat{\mathbf{s}}) & =\int_{0}^{\infty} d t t \Pi(Z, t ; \hat{\mathbf{s}}) .
\end{aligned}
$$

In the Figure 5 the mean arrival times $\tau_{\text {Sch }}$ and $\tau(\hat{\mathbf{s}})$ at detector location $Z=0$ have been plotted versus mass for free fall from rest $\left(k_{0}=0\right)$. The parameters of the initial wavepacket have been chosen as $z_{c}=8 \sigma_{0}$ and $\sigma_{0}=10 \mu \mathrm{m}$, $m_{\mathrm{n}}=1.67 \times 10^{-27} \mathrm{~kg}$ and $t_{\mathrm{ref}}=\frac{2 m_{n} \sigma_{0}^{2}}{\hbar}=3.161 \mathrm{~ms}$. One sees that the mean arrival time is mass-dependent, thus signifying a violation of WEQ. Inclusion of spin causes the mean arrival time to decrease with a small magnitude compared to the case of no spin. Hence, in comparison with the results for the case considering the effect of particle statistics without spin-dependence displayed in the figure 3, we find that the contribution of including spin explicitly at the level of the probability current leads to a much smaller modification of the mean arrival time values. However, here (Figure 5) again we find that the mass dependence of the mean arrival time vanishes for large mass, signifying emergence of WEQ in the classical limit. 


\section{CONCLUSIONS}

In this work we have investigated the effect of particle statistics on the arrival time distribution of Gaussian wave packets. We have employed the probability current approach [15] for computing the arrival time distribution for a system of two particles. The probability current approach provides an unambiguous definition [15, 23] of the arrival time of quantum particles, and also leads to a proper classical limit 22] of quantum dynamics. The single-particle arrival time distributions using the probability current approach have been studied earlier in the literature for the cases of free evolution, leading to the interesting effect of the spin-dependence [16, 18, 19] of arrival time. It was also observed earlier that the mean arrival time for particles freely falling under gravity acquires mass-dependence, thus signifying violation of the weak equivalence principle of quantum mechanics [12, 13]. Such violation stems essentially from the spread of propagating wavepackets that is mass-dependent.

In the present analysis we have shown for the first time that symmetrization or asymmetrization of a two body wavefunction leads to modification of the arrival time distribution even for freely propagating wavepackets. The impact of particle statistics on the arrival time distribution is observed for initially overlapping Gaussian wavepackets. For the case of particles falling freely under gravity, the mean arrival time acquires mass dependence signifying violation of WEQ. The magnitude of violation is seen to be different for MB, BE and FD statistics as a consequence of the difference in the mass dependence of the mean arrival time for the case of different statistics. We have also compared such a violation under the influence of particle statistics with the violation of WEQ obtained through the explicit introduction of particle spin in the probability current. The modification of the mean arrival time in the former approach is shown to be larger than in the latter for the case of Gaussian wavepackets considered here. However, in both the approaches, WEQ emerges smoothly in the limit of large mass. It may be noted that each of the three statistics gives a different mean arrival time in the large mass limit. Such difference stems essentially from the difference in the position expectation value and the spread of the wave packets for the respective statistics, and may be viewed as a remnant effect of particle statistics in the classical limit.

We conclude by observing that the effect of particle statistics in different quantum phenomena is regarded to be of much importance motivating newer tests beyond traditional arenas [26]. Our approach of studying the influence of particle statistics on the arrival time distribution provides an independent manifestation of the fundamental quantum property of indistinguishability. Moreover, the analysis of transit and flight times [4, 14] is a key ingredient in experiments involving Bose condensates which form a major avenue of revealing the effect of quantum statistics. The results reported in the present paper using symmetric Gaussian wavepackets furnish an example of the effect of particle statistics on the arrival time distribution. Though the magnitude of the difference in observable quantities resulting from different statistics, as computed in the present work may not be high enough for direct experimental detection, they provide an in principle signature of differential violation of WEQ for different statistics. More elaborate calculations are needed using different sets of parameters, and various forms of wavepackets such as asymmetric [18] or non-Gaussian [13] ones in order to raise the possibility of observational verification of such effects.

Acknowledgements: ASM and DH acknowledge support from the project SR/S2/LOP-08/2013 of DST, India. DH acknowledges support from the Centre for Science, Kolkata.

[1] M. H. Anderson et al., Science 269, 198 (1995).

[2] M. Griener et al., Nature 415, 39 (2002).

[3] J. I. Cirac and P. Zoller, Nat. Phys. 8, 264 (2012).

[4] T. P. Polak and T. A. Zaleski, Phys. Rev. A 87, 033614 (2013).

[5] P. R. Holland 1993, The Quantum Theory of Motion (Cambridge: Cambridge University Press)

[6] D. M. Greenberger and Overhauser A W Rev. Mod. Phys. 51 (1979) 43;

D. M. Greenberger Ann. Phys. 47 (1968) 116;

D. M. Greenberger Rev. Mod. Phys. 55 (1983) 875

[7] R. Colella, A. W. Overhauser and S. A. Werner Phys. Rev. Lett. 34 (1975) 1472

[8] A. Peters, K. Y. Chung and S. Chu Nature 400 (1999) 849

[9] C. Lämmerzahl Gen. Rel. and Grav. 28 (1996) 1043

[10] L. Viola and R. Onofrio Phys. Rev. D 55 (1997) 455

[11] P. C. W. Davies Class. Quantum Grav. 21 (2004) 2761

[12] Md. M. Ali, A. S. Majumdar, D. Home and A. K. Pan Class. Quantum Grav. 23 (2006) 6493

[13] P. Chowdhury, D. Home, A. S. Majumdar, S. V. Mousavi, M. R. Mozaffari and S. Sinha Class. Quantum Grav. 29 (2012) 025010

[14] Md. M. Ali, A. S. Majumdar, D. Home, A. K. Pan, Phys. Rev. A 75, 042110 (2007); J. Viana Gomes, M. Belsley, D. 
Boiron, Phys. Rev. A 77, 026101 (2008); Md. M. Ali, A. S. Majumdar, D. Home, A. K. Pan, Phys. Rev. A 77, 026102 (2008); Md. M. Ali, H-S. Goan, J. Phys. A: Math. Theor. 42, 385303 (2009).

[15] R. S. Dumont and T. L. Marchioro II, Phys. Rev. A 47, 85 (1993); C. R. Leavens, Phys. Lett. A 17827 (1993); Phys. Rev. A 58, 840 (1998); V. Delgado, Phys. Rev. A 591010 (1999).

[16] P. R. Holland Phys. Rev. A 60 (1999) 4326; Ann. Phys. 12, 446 (2003); P. Holland and C. Philippidis, Phys. Rev. A 67, 062105 (2003).

[17] J. Finkelstein, Phys. Rev. A 59, 3218 (1998).

[18] Md. M. Ali, A. S. Majumdar, D. Home, S. Sengupta, Phys. Rev. A 68, 042105 (2003).

[19] W. Struyve, W. D. Baere, J. D. Neve, S. D. Weirdt, Phys. Lett. A 322, 84 (2004).

[20] V. Zelevinsky 2011, Quantum Physics: From Time-Dependent Dynamics to Many-Body Physics and Quantum Chaos (Weinheim: Wiley-VCH).

[21] S. V. Mousavi and S. Miret-Artés Phys. Scr. 90 (2015) 025001

[22] Md. M. Ali, A. S. Majumdar, and A. K. Pan, Found. Phys. Lett. 19, 723 (2006).

[23] W. R. McKinnon and C. R. Leavens, Phys. Rev. A 51, 2748 (1995); C. R. Leavens, Phys. Rev. A 58, 840 (1998).

[24] M. Daumer, in Bohmian Mechanics and Quantum Theory: An Appraisal, edited by J. T. Cushing, A. Fine, and S. Goldstein (Kluwer, Dordrecht, 1996).

[25] A. D. Baute, I. L. Egusquiza and J. G. Muga, Phys. Rev. A 65 (2002) 032114.

[26] D. English, V. V. Yaschuk, D. Budker, Phys. Rev. Lett. 104, 253604 (2010). 